\title{
STRATEGIEN ZUR TRANSFORMATION VON DIENSTLEISTUNGEN AUF INFORMATIONSTECHNOLOGIE AM BEISPIEL DER WIRTSCHAFTSPRÜFER IN DER EUROPÄISCHEN UNION
}

\author{
Richard Wittsiepe ${ }^{1}$, Romualdas Ginevičius ${ }^{2}$ \\ ${ }^{1}$ International Consulting Firm C PA. E-mail: richard@ cpa.com ${ }^{2}$ Vilnius \\ Gediminas Technical University, Sauletekio ai 11, LT-2040 Vilnius, Lithuania \\ E-mail: rector@adm.vtu.lt
}

Received: 21 October, 2002

\begin{abstract}
Strategy of Based on Information Technologies Services' ${ }^{1}$ Transformation: Case Study of European Union's Analysts.

Abstract. Currently implementation of informational society is seen by European Commission as important factor, which could lead to improving of compatriots' living standards. Despite number of internet users exceeds 50 millions, it still makes only as much as one third of that number in USA. Consequently questions related to information technologies Services' transformation strategy have become of prime importance. Presented paper deals with the issue on basis of Web Trust Assurance Service methodic proposed by American Institute of Certified Public Accountants (AICPA). This new system embraces four following areas: needs of clients, mega trends, informational technologies and auditors' competence. Analysis shows that contemporary financial statement audit system doesn't meet requirements of investors, creditors and other users. At first, it is not of prime importance. The second, auditors are expected to perform their tasks only formally. The third, notion of auditor's independence remains obscure. The new audit system is more oriented to informational technologies. It includes determination of informational aspects, which are necessary for performance of audits' functions, also determination of potential clients' groups, development of new norms, criteria and etc. In this system the main role has been played by auditors. In the paper it has been emphasized that adoption of the system in Baltic countries would accelerate integration into European Union
\end{abstract}

Keywords: information technologies' services, strategy, auditing system.

\section{Einleitung}

Die Kommission der Europäischen Union [1] sieht in der sich entwickelnden Informationsgesellschaft das Potential, Europa in eine Gesellschaft und Wirtschaft zu verwandeln, in der fortschrittliche Technologien mit dem Ziel genutzt werden, die Lebens- und Arbeitsbedingungen aller Bürger zu verbessern.

Wenn Europa das sich bietende Potential nutzt, verspricht die Informationsgesellschaft eine Reihe von Vorteilen. Dazu gehört ein höherer Lebensstandard, und die Möglichkeit für Unternehmen, sich an neuen Wachstumsmärkten zu beteiligen, Veränderungen in der Art und Weise, wie bestehende Produkte und Dienstleistungen mit Produktivitätszuwächsen produziert und geliefert werden.

Die Volkswirtschaften der entwickelten Industrieländer erleben derzeit einen umfangreichen technologischen Transformationsprozeß. Dieser Proze $ß$ wird sichtbar an der raschen Zunahme des Anteils von Informationstechnologien in allen Bereichen. Das Tempo dieser Veränderungen ist die Folge enormen technischen Fortschritts, der sich an der Produktion von Informa- tionstechnologie (IT) Ausrüstung ablesen läßt, zu erkennen an dem starken Rückgang der Preise für ITGüter, die sich für Ausrüstungsinvestitionen (im Vergleich zu Verbraucherpreisen) von über $40 \%$ in den USA und ungefähr 30\% in Europa bewegen. Dieser Prozeß wird sich bei einem wachsenden Anteil von IT an der Kapitalbildung aller Voraussicht nach beschleunigen.

Ein weiteres Kennzeichen dieser Entwicklung ist das Anwachsen des elektronischen Handels in Europa. Elektronischer Handel, vor wenigen Jahren noch fast völlig unbekannt, verzeichnet heute starkes Wachstum. Es wird angenommen, daß elektronischer Handel in Europa von 17 Milliarden US-Dollar (USD) Ende 1999 auf etwa 360 Milliarden USD im Jahr 2003 anwachsen wird und sich damit jährlich etwa verdoppelt. Elektronischer Handel zwischen Unternehmen wird dabei etwa 90\% des gesamten E-Handels ausmachen und bietet damit ein sehr viel größeres Potential als der Handel zwischen Unternehmen und Verbrauchern. Über seinen direkten, in Einnahmen meßbaren Einfluß hinaus, wird E-Handel Unternehmensprozesse und Organisationsmodelle in großem Umfang verändern. 
Die Verbreitung des Internet in Europa - ein aussagekräftiger Indikator für die allgemeine Entwicklung der Informationsgesellschaft - hat einen riesigen Sprung nach vorn gemacht. Europa ist jetzt der am schnellsten wachsende Markt für die Internet-Entwicklung. Die Zahl der Internet-Nutzer betrug Ende 1999 etwa 50 Millionen und wird sich bis 2003 voraussichtlich verdoppeln. Trotzdem erreicht die Internet-Verbreitung in Europa nur ein Drittel der Verbreitungsrate in den USA.

\section{Die Initiative eEurope}

Die Initiative eEurope wurde am 8. Dezember 1999 mit dem Beschluß der Mitteilung 'eEurope - Eine Informationsgesellschaft für alle' durch die Europäische Kommission eingeleitet [2].

Ziel dieser Initiative ist es, die Akzeptanz der digitalen Technologien in ganz Europa zu fördern und dafür zu sorgen, daß alle Europäer die für die Nutzung dieser Technologien erforderlichen Fähigkeiten erwerben. Sie spielt eine zentrale Rolle in der Agenda für eine wirtschaftliche und soziale Erneuerung Europas, welche die Kommission für die Sondertagung des Europäischen Rates in Lissabon ausgearbeitet hat.

Den Anstoß zu der Initiative gab die Erkenntnis, daß die digitalen Technologien zum entscheidenden Motor für Wirtschaftswachstum und Beschäftigung geworden sind. Es mehren sich die Anzeichen dafür, daß eine 'neue Ökonomie' in Entstehung begriffen ist, deren Entfaltung in erster Linie vom Internet vorangetrieben wird. Europa hat eher langsam auf die mit diesem Prozeß einhergehenden Chancen und Herausforderungen reagiert, was insbesondere darauf zurïckzuführen ist, daß Europa noch immer den Denkmustern der traditionellen Industriewirtschaft verhaftet ist.

Der Europäische Rat in Lissabon wollte dies ändern. Durch die Bekräftigung konkreter politischer Maßnahmen wurde ein deutliches Signal dafür geben, daß die europäische Politik entschlossen ist, Europa in einen dynamischen und wettbewerbsfähigen Wirtschaftsraum zu verwandeln. Damit diese Transformation gelingen kann, muß vor allem die Dynamik des Marktes und des Privatsektors genutzt werden. Die eEurope-Initiative soll diese Entwicklung anregen.

Von diesem Transformationsprozeß sind alle Berufszweige betroffen. Wie eine solche Transformation aussehen kann, zeigt der Berufsstand der Wirtschaftsprüfer. $\mathrm{Zu}$ den traditionellen Dienstleistungen dieses Berufsstand zählt die gesetzliche Prüfung von Jahresabschlüssen der Unternehmen. Mit der Prüfung und dem Bestätigungsvermerk eines Wirtschaftsprüfers wird den Empfängern der Jahresabschlußinformationen eine Aussage über die Übereinstimmung der Zahlen mit gesetzlichen Vorschriften zur Verfügung gestellt. Diese Aussage kann die Anlageentscheidung von Kapitalgebern wesentlich beeinflussen.

Bei der Transformation der traditionellen Dienstleistung wird ein Umstand deutlich, der in den Berichten der Kommission der Europäischen Union angesprochen wird, nämlich der Rückstand der Europäischen Union gegenüber den USA. Die aktuellen Ansätze zur Transformation der Dienstleistungen der Wirtschaftsprüfer haben ihren Ursprung in den USA. Dieses Beispiel gibt auch einen Eindruck vom zeitlichen Vorsprung der USA, denn die Vorüberlegungen begangen dort schon im Jahre 1993, während vergleichbare Ansätze innerhalb der Europäischen Union erst jetzt forciert werden.

\section{WebTrust Assurance Service als Internet- Dienstleistung der Wirtschaftsprüfer}

Der WebTrust Assurance Service wurde im Jahre 1997 durch das American Institute of Certifies Public Accountants (AICPA) [3] der Öffentlichkeit vorgestellt. Es handelt sich um die erste Dienstleistung, die der Entwicklung des Internets Rechnung trägt und versucht, ein Konzept für die Transformation der traditionellen Dienstleistungen auf die Anforderungen der Informationsgesellschaft $\mathrm{zu}$ entwickeln. Das AICPA versteht sein Angebot als weltweites Angebot und hat insbesondere in Bezug auf eine mögliche Übernahme der Dienstleistung in die Europäische Union die Assurance Services im Detail an grundlegende Vorschriften der Europäischen Union, darunter z.B. Datenschutz- und Konsumentschutzvorschriften, angepaßt. Verschiedene berufsständische Dachorganisationen, insbesondere aus den angelsächsischen Ländern, darunter England, haben den WebTrust Assurance Service übernommen und es ist nicht auszuschließen, daß sich weitere Länder in der EU sich dieser Entwicklung anschließen werden.

\subsection{Grundlagen der Assurance Services}

\subsubsection{Das AICPA Special Committee on Assurance Services}

Die Gründung des Special Committee on Assurance Services geht zurück auf eine dreitägige Konferenz, die das AICPA im Jahre 1993 unter der Bezeichnung „Audit/Assurance-Konferenz" in Santa Fe veranstaltete. Gegenstand der Konferenz sollte eine breite Diskussion über die aktuelle Situation der Audit und 
Attest Services als klassische Dienstleistung des Berufsstandes sein.

Es war zu diesem Zeitpunkt nicht mehr zu übersehen, daß der Berufsstand mit seinen Standarddienstleistungen zunehmend im Markt auf Probleme traf. Neben seit Jahren rückläufigen bzw. stagnierenden Umsätzen zeigte sich dieser Negativtrend auch in einer allgemeinen Unzufriedenheit der Empfänger von Wirtschaftsprüfer-Dienstleistungen mit dem Dienstleistungsangebot [4|.

Diesen zunehmenden Problemen und negativen Äußerungen im Markt standen keine systematisch gewonnenen Analysen über die Gründe zur Verfügung. Ebenfalls gab es kein Angebot möglicher Gegenstrategien des Berufsstandes.

Die Santa Fe-Konferenz, deren Teilnehmer aus dem Kreis der Berufsangehörigen, Universitäten, Behörden und anderen interessierten Kreisen aus den USA und Kanada stammten, sollte die Probleme auflisten, analysieren und einen Ausgangspunkt für die Entwicklung von Gegenstrategien bieten.

Als erstes Ergebnis der Santa Fe-Konferenz wurde das Special Committee on Assurance Services (im Folgenden auch Assurance Committee genannt) gegründet und dessen Aufgabenstellung definiert [5]. Das Assurance Committee sollte als „Leadership Team" einen strategischen Plan zur Ausweitung der Assurance Services des Berufsstandes entwickeln. Die Erweiterung dieser Form von Dienstleistungen sollte sich an den Wünschen der Kunden orientieren. Die Ausweitung der Assurance Services wurde als Kernpunkt der Gegenstrategie zu einer negativen Marktentwicklung festgelegt.

\subsubsection{Die Vorgehensweise des Special Committee on Assurance Services}

Rückwirkend betrachtet stellt sich der Zeitraum zwischen der Santa Fe-Konferenz im Jahre 1993 über die erste konstituierende Sitzung des Assurance Committee im November 1994 [6] bis zur öffentlichen Präsentation des WebTrust Service als erste der neuen Dienstleistungen einschließlich des Angebots von Schulungskursen für die Berufsangehörigen im Herbst 1997 mit insgesamt vier Jahren relativ kurz dar [7].

Die erste Phase zwischen der Santa Fe-Konferenz und der konstituierenden Sitzung des Assurance Committee im November 1994 diente der Erfassung der in der Literatur bereits veröffentlichten Studien und Analysen über die Entwicklung des Berufsstandes und seiner Dienstleistungen sowie der Aufstellung einer ersten Auswertung [8].
Auf dieser Grundlage wurden eigene Untersuchungen angestellt, die aber nicht den Anspruch einer empirischen oder repräsentativen wissenschaftlichen Untersuchungen erhoben. Dies hätte nach Ansicht des Assurance Committee zu viel Zeit in Anspruch genommen und es wurde darauf verzichtet.

Da das Assurance Committee zur Entwicklung neuer Dienstleistungen die zukünftige Marktentwicklung beurteilen und eine Strategie entwickeln sollte, wurde auf bestehende Trendanalysen zurückgegriffen und diese wurden ergänzt durch eigene Interviews und Beobachtungen sowie einen Erfahrungsaustausch der Mitglieder des Assurance Committee.

Folgende Bereiche wurden betrachtet:

\section{Kundenbedürfnisse \\ 2. Megatrends \\ 3. Informationstechnologien \\ 4. Kompetenzen der Wirtschaftsprüfer}

\subsection{Identifikation neuer Servicefelder}

Bei der Bestimmung neuer Servicefelder wurden durch das Assurance Committee drei Ziel vorgaben gesetzt:

1. Entwicklung eines neuen Konzeptes für berufsbezogene Services unter der Bezeichnung Assurance Services als Basis für neue geschäftliche Möglichkeiten mit gleichzeitigen Konzeptentwicklungen zur Begrenzung des Haftungsrisikos, zur Unabhängigkeit des Wirtschaftsprüfers sowie zur Relevanz des Service aus der Sicht möglicher Nutzer.

2. Bei der Suche nach neuen Services soll der Schwerpunkt auf solche Bereiche gelegt werden, die eine Ergänzung zu bestehenden Services bilden können mit der Möglichkeit, diese vorhandenen Mandanten anzubieten. Die Services sollen eine Mischung aus Elementen bestehender Services mit neuen Ansätzen ergeben und müssen sowohl ein langfristig positives Marktpotential bieten als auch kurzfristig im Markt einführbar sein. Bei der Konzeption sollen einige der neuen Services besonders auf den Einsatz durch kleinere und mittelständische Wirtschaftsprüfer-Kanzleien zugeschnitten werden.

3. Es soll ein permanenter Prozess der Entwicklung neuer Services installiert werden, der aus Marktanalysen neue Servicemöglichkeiten identifiziert, Vorschläge erarbeitet und Entwürfe vorstellt. Alle Services sollen auf Kundenbedürfnisse zugeschnitten werden. Bestehende Services sollen einem kontinuierlichen Anpassungsprozess an sich verändernde Marktbedingungen ausgesetzt werden. 


\subsection{Probleme der traditionellen Dienstleistung Financial Statement Audit}

Das Ergebnis der Überlegungen kann in einer Kernaussage zusammengefaßt werden:

Der Financial Statement Audit erfüllt nicht mehr das Informationsbedürfnis von Investoren, Gläubigern und anderen Nutzern.

Nachfolgend sind Erklärungsansätze für die Kernaussage angeführt [9]:

1. Das derzeitige Modell des Financial Statement Audit wird zunehmend für die Zielgruppe irrelevant.

2. Von einem Wirtschaftsprüfer wird mehr erwartet als nur die Abgabe einer formalen Erklärung über Financial Statements.

3. Der Begriff der Unabhängigkeit des Wirtschaftsprüfers muss dem Nutzer klarer vermittelt werden.

4. Ein Wirtschaftsprüfer wird in der Praxis von Haftungsfragen gehemmt.

Informationen aus dem Rechnungswesen dienen traditionell als Unterstützung zur Entscheidungsfindung. Veränderungen im Entscheidungsprozeß des Managements führen zu einer permanenten Erhöhung der Anforderungen an das Rechnungswesen zur Bereitstellung entscheidungsrelevanter Informationen mit einer Tendenz zu aktuellen Informationen. Ein testierter Jahresabschluß verliert in diesem Kontext zunehmend an Bedeutung, da er eine begrenzte Aussage über vergangensheitsbezogene Informationen enthält, die nicht mehr dem Informationsbedürfnis der Entscheider entspricht.

Das Informationsbedürfnis der Interessenten wird durch alternative Informationsangebote erfüllt, z. B. durch externe Analysten, Banken oder Finanzdienstleister, aber auch durch komplexe Informationssysteme im Unternehmen selbst. Mit dem Aufkommen alternativer Informationsangebote verliert der Jahresabschluß seine Bedeutung in Bezug auf die Informationsfunktion.

\subsection{Wettbewerbsvorteile des CPA}

Trotz der aufgezeigten negativen Einschätzungen über die Wettbewerbsfähigkeit des Financial Statement Audits konnte ein Merkmal identifiziert werden, das von den Marktteilnehmern als Stärke des CPA gekennzeichnet und als positives Wettbewerbsmerkmal bezeichnet wurde:

Im Vergleich zu anderen Berufsgruppen genießt der Berufsstand der CPA einen Vertrauensvorschuss. 16
Dieses Vertrauen in die Neutralität und Kompetenz seiner Aussagen grenzt ihn positiv von anderen Berufsgruppen $a b$ und wird besonders von kleinen und mittleren Unternehmen als Qualitätsmerkmal genannt [10].

Dieses Qualitätsmerkmal wurde als zentraler Ansatzpunkt zur Entwicklung neuer Dienstleistungen gewählt. Damit sollte erreicht werden, daß der CPA im Wettbewerb mit anderen Berufsgruppen und Anbietern von Informationen einen Wettbewerbsvorteil realisieren und im Markt vermitteln kann.

\section{Bedeutung der Informationstechnologie}

\subsection{Strategieempfehlungen des AICPA Future Issue Committee}

In allen Prognosen wird der Informationstechnologie [11] eine große Bedeutung beigemessen. Das AICPA Future Issues Committee faßte die Rolle der Informationstechnologie wie folgt zusammen [12]:

1. Angesichts der technologischen Entwicklung einer Gesellschaft, die zunehmend auf Informationen basiert, werden sich Wirtschaftsprüfer mehr an der Informationstechnologie beteiligen müssen.

Um in einem solchen Umfeld konkurrenzfähig zu bleiben, werden Unternehmen technologische Entwicklungen wie Expertensysteme einsetzen. Der daraus resultierende Bedarf an Dienstleistungen für das Informationsmanagement eines Unternehmens muß auch durch Angebote des Wirtschaftsprüfers bedient werden.

2. Während die Entwicklung zur Informationsgesellschaft im Gange ist, wird der Berufsstand auf neue Schwierigkeiten im Markt und Wettbewerb stoßen, aber auch neue Marktchancen erhalten, die durch den Anstieg der Informationsmenge entstehen. Die Attest Function muß auf eine wesentlich breitere Informationspalette ausgedehnt werden als bisher und darf sich nicht auf den Financial Statement Audit beschränken.

3. Die Erwartungslücke aus dem Anspruch der Öffentlichkeit an das Ergebnis der Tätigkeit des CPA und dessen Fähigkeit, diese Erwartungen zu erfüllen, wird bestehen bleiben bei gleichzeitiger Erhöhung der öffentlichen Erwartungshaltung an ein höheres Leistungsniveau der CPA.

4. CPA werden zunehmend Dienstleistungen anbieten und erbringen, die sich auf zukünftige Entwicklungen beziehen. Der relative Anteil und die Bedeutung der zukunftsorientierten finanziellen und nichtfinanziellen 
Informationen wird zunehmen und die Bedeutung der vergangenheitsbezogenen Financial Statements abnehmen [ 13].

Als Folge sollte die Attest Function auf eine breitere Basis erweitert werden. Möglicherweise führt diese Verbreiterung zu erhöhten Haftungsrisiken, zu höheren Qualitätsanforderungen und damit zu höheren Kosten zur Aufrechterhaltung der Dienstleistungsqualität mit der Folge, daßsich nur wenige Priifungsgesellschaften an dem erweiterten Dienstleistungsangebot beteiligen können oder wollen.

Die Strategieempfehlung des AICPA Future Issue Committee setzt sich aus folgenden Schritten zusammen:

1. Ermittlung der Informationsarten, fur die wahrscheinlich eine Attest Function erforderlich sein wird.

2. Ermittlung potentieller Nutzer.

3. Entwicklung neuer Normen, Kriterien und Richtlinien für neue Bereiche der Attest Function.

4. Prüfung der Vermarktungsmöglichkeiten.

5. Schaffung eines Konsens innerhalb des Berufsstandes für die Übernahme neuer Attest Functions.

Das Assurance Committee ist im Wesentlichen der Strategieempfehlung des AICPA Future Issue Committee gefolgt. Die aufgezeigte Gefahr einer Beschränkung neuer Services auf große und kapitalstarke Prüfungsgesellschaften sollte durch den ausdrücklichen Einbezug der kleinen und mittleren Kanzleien in die Konzeption neuer Services durch die Zielvorgaben des Assurance Committee verhindert werden.

\subsection{Die Rolle des Wirtschaftsprüfers in der Informationsgesellschaft}

Vor der Bestimmung neuer Dienstleistungsbereiche, die vor dem Hintergrund der prognostizierten Trends einen Mehrwert bieten könnten, wurden die Rolle des Wirtschaftsprüfers in der Informationsgesellschaft untersucht und die Voraussetzungen betrachtet, unter denen eine Nachfrage nach einer Attest Function grundsätzlich entstehen kann [14].

Nach Elliot lösen folgende Voraussetzungen eine wirtschaftliche Nachfrage nach Attest Functions aus [15]:

1. Wenn Nutzer Informationen benötigen, gibt es einen Interessenkonflikt zwischen dem Ersteller und dem Nutzer der Informationen.

2. Die Informationen müssen überprüfbar sein.

3. Das Kosten-Nutzen-Verhältnis für die Attest Function ist günstig.
$\mathrm{Zu}$ einem vergleichbaren Ergebnis kommt William $\mathrm{R}$. Kinney (Universität Texas):

Danach kann mit der Bestätigung eines Jahresabschlusses durch einen Prüfer für den Inhaber (eines Unternehmens) ein Mehrwert nur dann entstehen, wenn der Nutzer der Bestätigung an drei Dinge glaubt [16]:

1. Die auf Grundlage von GAAP erstellten Informationen sind für ihn wertvoll.

2. Der Wirtschaftsprüfer ist in der Lage, wesentliche Abweichungen von den GAAP festzustellen.

3. Der Wirtschaftsprüfer ist vertrauenswürdig genug und wird alle wesentlichen Abweichungen von GAAP berichten.

Die Cohen-Kommission hat die Rolle des Wirtschaftsprüfers wie folgt beschrieben [17]:

„Es gibt eine allgemeine Übereinstimmung in mehreren Aspekten hinsichtlich des Nutzens eines geprüften Abschlusses. Der wichtigste Aspekt ist, daß geprüfte Abschlüsse ein Mittel für die Bestätigung oder die Korrektur von Informationen darstellen, die der Markt zu einem früheren Zeitpunkt erhalten hatte. Geprüfte Abschlüsse sind eine effektive Hilfe, die Effizienz des Marktes zu gewährleisten, indem die Lebensdauer ungenauer Informationen begrenzt wird oder indem vor ihrer Verbreitung abgeschreckt wird."

Das Institute of Chartered Accountants von England und Wales kam als Ergebnis einer Untersuchung aus dem Jahre 1991 zu dem Schluß, dass für viele Nutzer der Abschlußprüfer als ein Wächter gewisser Ideale oder Handelsbräuche gesehen wird, die nicht unbedingt auf den Abschluß allein begrenzt sind [18].

Die Cohen-Kommission hat bei der Betrachtung der zukünftigen Rolle des Abschlußprüfers den Schluß gezogen, dass die traditionelle Verbindung von unabhängigen Wirtschaftsprüfern mit Jahresabschlüssen ein veraltetes und begrenztes Konzept ist.

Das sich verändernde Geschäfts- und Investitionsumfeld erfordert eine flexiblere und zeitgemäßere Art der Verbindung und die Abschlußprüfungsfunktion sollte sich in diese Richtung entwickeln.

Zusammenfassend kann als gemeinsames Ergebnis der angeführten Untersuchungen festgestellt werden, daß die Zukunft der Abschlußprüfung in einer Erweiterung der Informationen gesehen wird, die im Rahmen einer Abschlußprüfung betrachtet werden sollen.

\subsection{Neue Formen der Berichterstattung - Permanentes Berichtswesen}

Eine Möglichkeit, die aufgrund der Informations- 
technologie als wahrscheinlich angesehen wird, ist der Übergang zu einer kontinuierlichen Berichterstattung.

Die Cohen-Kommission führte dazu an, daß ,die Beziehungen zwischen einem Wirtschaftsprüfer und einem Unternehmen meist ständige Beziehungen sind. Der Trend bei der Offenlegung von Finanzinformationen geht auch in die Richtung eines ständigen Informationsflusses. Zu Zeiten, als ein Jahresabschluß die Hauptinformationsquelle finanzieller Informationen darstellte, war eine Prüfung der Abschlüsse einmal im Jahr ausreichend, bei einem ständigen Informationsfluß reicht dies nicht mehr aus."

Die Stock Exchange Commission (SEC) beurteilte die Frage der Berichterstattung über Datenbanken und kam zu dem Ergebnis, daß

„die Fähigkeit des Computers, Daten zusammenzufassen, aufzuspalten und wieder zusammenzufassen, nicht bedeutet, daß die Nutzer nur detaillierte Daten haben wollen. Die Technik ermöglicht es den Nutzern der Daten, alternative und multiple Zusammenfassungen ihrer eigenen Wahl durchzuführen, während ihnen bisher ein festes Schema vorgegeben wurde."

In der Diskussion über neue Formen der Berichterstattung wurde auch die Frage aufgeworfen, ob für den Fall ständiger Online Verfügbarkeit von Informationen überhaupt ein Geschäftsbericht veröffentlicht werden sollte [19].

Betrachtet man die Bandbreite der dargestellten Meinungen über die Zukunft der Abschlußprüfung und die Rolle des Abschlußprüfers, ergeben sich unterschiedliche Ansatzpunkte.

Die Schlußempfehlung des Mc Farlane-Committee bestand darin, daß eine Prüfermeinung, die in einem einzigen Ja-oder-Nein-Satz endet, die Komplexität der Erstellung von Abschlüssen nicht angemessen darstellt und deshalb anstatt einer stereotypen Bestätigung in der Form eines Kommentars über die materiellen Punkte im Rahmen einer Prüfung berichtet werden sollte [20].

\section{Zusammenfassendes Ergebnis}

Die Ergebnisse aus den Vorüberlegungen des Assurance Committee sind in einem Bericht zusammengefaßt, der sich mit der Zukunft der Prüfung vergangenheitsbezogener Abschlüsse auseinandersetzt [21].

In ihm werden die Auswirkungen der Veränderungen bezüglich des Bedarfs an Abschlußprüfungen im Markt sowie verschiedene andere externe Faktoren, darunter insbesondere die Auswirkungen der Informationstechnologie, betrachtet.
In dem Bericht wird auch die Frage diskutiert, ob das bestehende Abschlußprüfungssystem in der aktuellen Form überleben wird.

Das Assurance Committee kommt nach dieser Betrachtung $\mathrm{zu}$ dem Ergebnis, daß derzeit ein bedeutender Paradigmenwechsel im Gang ist, der sowohl die Informationsarten selbst, mit denen Abschlußprüfer konfrontiert werden, als auch die Art und Weise, wie das Auftragsverhältnis definiert wird, betrifft.

Der Wandel kann wie folgt beschrieben werden:

\begin{tabular}{|l|l|}
\hline Altes Muster der Abschlußprüfung & Neues Muster der Abschlußprüfung \\
\hline $\begin{array}{l}\text { Ein Satz Jahresabschlüsse, ergänzt } \\
\text { durch einen Jahresprüfungsbericht }\end{array}$ & $\begin{array}{l}\text { Ein Satz finanz- und nicht- } \\
\text { finanzbezogener Echtzeit- } \\
\text { Informationen, ergänzt durch } \\
\text { kontinuierliche Assurance-Services } \\
\text { (für Kunden und möglicherweise für } \\
\text { die Öffentlichkeit) }\end{array}$ \\
\hline
\end{tabular}

Auf dieser Grundlage definiert das Assurance-Committee einen Assurance Service wie folgt [22]:

Independent professional services that improve the quality of information, or its context, for decision makers.

Eine Übersetzung könnte wie folgt lauten: Assurance Services sind „unabhängige professionelle Dienstleistungen, die die Qualität der Informationen oder ihren Zusammenhang für Entscheider verbessern."

\subsection{Mehrwert eines Assurance Service}

Auf eine kurze Formel gebracht könnte das Ziel eines Assurance Service darin gesehen werden, Entscheidern bessere Informationen zur Verfügung zu stellen, als in der Situation ohne Assurance Service zur Verfügung stehen würde. Aus der Sicht eines Entscheiders wäre ein Vergleich seiner Informationssituation mit und ohne Assurance Service anzustellen. Da ein Assurance Service nicht durch gesetzliche Vorschriften oder gesellschaftliche Traditionen zwingend vorgeschrieben ist, wird der Service nur dann gekauft, wenn er dem Erwerber einen höheren Wert verspricht im Verhältnis zu den Kosten.

Der Mehrwert einer verbesserten Information als Ziel der Dienstleistung kann in zwei Aspekten in Erscheinung treten:

1. Der Entscheider erhält wichtige Informationen über sein Unternehmen, die ihm vorher nicht bekannt oder bewußt waren. Interner, z.B. auf das Unternehmen bezogener, Mehrwert.

2. Der Entscheider kann aber auch ein Kunde des Auftraggebers eines Assurance Service sein. Der Mehrwert besteht in dieser Konstellation in der 
Verbesserung der Informationssituaton des Kunden.

Der Wirtschaftsprüfer erbringt in der zweiten Funktion einen Mehrwert als Informationsmittler zwischen Unternehmen und deren Kunden.

Die Konzeption solcher Assurance Services setzt zwei Dinge voraus:

1. Ein Verständnis der Entscheidungsprozcsse.

2. Kenntnis der Relevanz der Informationen, die bei diesen Prozessen verwendet werden.

Mit dem WebTrust Audit wird die Funktion des Informationsmittlers zwischen Unternehmen und seinen Kunden im Rahmen von Electronic Commerce (EC) Anwendungen ausgefüllt. Für die Untersuchung der WehTrust Audit Procedures ist deshalb eine Betrachtung der Entscheidungsprozesse und Informationsarten notwendig, die für den Kunden des beauftragten Unternehmens von Bedeutung sein können.

Entscheider sind die Endkunden eines Assurance Ser〉>ice. Sie sind diejenigen, die tatsächlich die Dienstleistungen verwenden, unabhängig davon, ob sie diese tatsächlich bezahlen.

Zur Ermittlung von Informationsbedürfnissen hat das Assurance Committee Umfragen potentieller Kunden durchgeführt, deren Ergebnis die nachfolgende Tabelle zeigt [23]:

\begin{tabular}{|l|l|}
\hline Kundenbedürfnis & Potentielle Kunden \\
\hline $\begin{array}{l}\text { Bessere Informationen über } \\
\text { Geschäftsrisiken }\end{array}$ & Vorstand, Management \\
\hline Informationen über Produktqualität & Einzelpersonen \\
\hline Leistungsmaßstäbe & Leitende Angestellte \\
\hline $\begin{array}{l}\text { Qualität der dem Vorstand } \\
\text { gemeldeten Informationen }\end{array}$ & Vorstand, institutionelle Anleger \\
\hline $\begin{array}{l}\text { Qualität der Prozesse und der } \\
\text { Kontrollen }\end{array}$ & $\begin{array}{l}\text { Vorstände, Leitende Angestellte, } \\
\text { Investoren }\end{array}$ \\
\hline $\begin{array}{l}\text { Informationen über die Ausführung } \\
\text { der strategischen Planung }\end{array}$ & Vorstände, institutionelle Anleger \\
\hline $\begin{array}{l}\text { Informationen über Leistung der } \\
\text { Regierung }\end{array}$ & Öffentlichkeit \\
\hline
\end{tabular}

Die Tabelle zeigt bestehende Informationsbedürfnisse zum Zeitpunkt der Interviews. Das Problem bei der Konzeption neuer Assurance Serx'ices besteht in der Prognose der Entwicklung zukünftiger Informationsbedürfnisse.

Die Trends sind zwar bekannt, aber wie tatsächlich die Entwicklungen der Informationstechnologie, Veränderungen der Unternehmensstrukturen hin zu virtuellen Organisationen, anwachsende Kapitalströme, zusätzliche Forderungen der Öffentlichkeit nach Rechenschaftslegung [24] sowie Veränderungen der Altersstruktur der Bevölkerung das Informationsbedürfnis beeinflussen werden, ist nicht vorhersehbar.
Dies ist ein Grund, weshalb Assurance Services als eine „flexible Dienstleistung" konzipiert sind. Der Anpassungsprozeß an sich verändernde Informationsbedürfnisse soll abgekürzt werden.

Alle vorgestellten neuen Dienstleistungen aus der Reihe der Assurance Services basieren auf den vorgenannten Konzept. Es handelt sich dabei neben dem WebTrust Assurance Service um die Dienstleistungen WebTrust-ISP (Internet Service Provider) und SysTrust (Systems Reliability).

\section{Schlußfolgerungen für die baltischen Staaten}

Die Initiative eEurope der Europäischen Union verfolgt u.a. die Zielsetzung, die Transformation der Wirtschaft auf die Anforderungen der Informationstechnologie zu unterstützen und den Rückstand gegenüber den USA aufzuholen. Betrachtet man das Beispiel der Transformation der WirtschaftsprüferDienstleistung, so wurden die wesentlichen Vorüberlegungen in den USA durchgeführt und sogar schon Anpassungen der US Standards an EU Vorschriften vorgenommen. Um auf diesem Gebiet einen Rückstand gegenüber den USA aufzuholen bedarf es im Prinzip der Schaffung der notwendigen gesetzlichen Grundlagen für die Anwendung der Dienstleistungen innerhalb der EU. Die Dienstleistung selbst muß nicht neu entwickelt werden, sie liegt bereits vor. Im Kern handelt es sich hierbei nicht nur um die Beschreibung einer neuen Dienstleistung, sondern um die Beschreibung eines Geschäftsmodells für einen ganzen Berufsstand unter dem Blickwinkel der Anforderungen der Informationsgesellschaft.

Solche Geschäftsmodelle liegen für eine Reihe von Berufsgruppen und Wirtschaftszweigen bereits vor. Die Entwicklungen auf dem Markt der Telekommunikation als Anbieter der Infrastruktur für den elektronischen Handel zeigen dies deutlich auf. Und genau an dieser Stelle besteht auch die große Chance der baltischen Staaten, denn es eröffnet sich die Möglichkeit, sofort diese neuen Geschäftsmodelle anzuwenden, es würde kein Rückstand gegenüber den derzeitigen Mitgliedsstaaten der EU entstehen. Ein Transformationsproze $\beta$ findet in den baltischen Staaten ohnehin statt, da die Strukturen der Planwirtschaft nicht weitergeführt werden können. Es wäre aber ein Fehler, diese planwirtschaftlichen Strukturen durch solche Geschäftsmodelle zu ersetzen, die jetzt im Rahmen der eEurope Initiative transformiert werden sollen. 


\section{Abkürzungsverzeichnis:}

AICPA - American Institute of Certified Public Accountants; CPA - Certified Public Accountants; EC Electronic Commerce; GAAP - General Accepted Accounting Standards; IT - Informationstechnologie; JoA - Journal of Assountancy; SEC - Stock Exchange Commission

\section{Literatur}

1. Mitteilung der Kommission der Europäischen Gemeinschaften: "Strategien für Beschäftigung in der Informationsgesellschaft", Brüssel, 4. Februar 2000.

2. Kommission der Europäischen Union: „Eine Informationsgesellschaft für alle. Zwischenbericht für den Sondergipfel des Europäischen Rates zum Thema Beschäftigung, Wirtschaftsreform und sozialer $\mathrm{Zu}-$ sammenhalt - für ein Europa der Innovation und des Wissens" Lissabon, 23./24. März 2000.

3. Siehe www.aicpa..org.

4. Assurance Committee:"Preliminary Research on the Future of Auditing", www.aicpa.org.

5. AICPA: "Origin of the Committee: The 1993 Audit/Assurance Conference", www.aicpa.org.

6. Assurance Committee: "The Committee's Approach", www.aicpa.org.

7. Korèto, Richard: „In CPAs we trust" in JoA December 1997, S. 62 ff.

8. Die Ergebnisse dieses Prozesses sind in drei Aufsätzen zusammengefasst: Elliot, Robert K.; Pallais, Don M.: „Are you ready for new Assurance Services?" in JoA June 1997, S. 47 ff. Elliot, Robert K.; Pallais, Don M.: „First know your market" in JoA July 1997, S. 56 IT. Elliot, Robert K.; Pallais, Don M.: „Build on your firm's strengths" in JoA August 1997, S. 53 ff.

9. Mednik, Robert: "Reinventing the Audit", JoA, August 1991.
10. Magill, Harry T; Previts, Gary J.; Robinson, Thomas R.: "The CPA Profession: opportunities, responsibilities, and services", S. 195 ff., 1998

11. Steed, Vai D.: „Must Technologies for all CPAs" in JoA March 1997, S. 59 ff.

12. AICPA Future Issues Committee: "Strategic Thrusts for the Future", May 1991.

13. Gelinas, Ulric J.; Sutton, Steve G; Oram, Allan E.: „Accounting Information Systems", 4th ed., Cincinnati 1998.

14. Siehe auch: Thomas, Joyce: „The Future - It is us; Special Report: CPA Vision 2011 and Beyond: Focus on the Horizon" in JoA December 1998, S. 23 ff.

15. Elliot, Robert K.: "The Third Wave Breaks on the Shores of Accounting", Accounting Horizon, Juni 1992.

16. Kinney, William: "Audit Litigation Research: Professional Help is Needed", Accounting Horizons, June 1994.

17. Commission on Auditors Responsibilities: Report, Conclusions and Recommendations, AICPA 1978.

18. The Institute of Chartered Accountants in England and Wales: "The Audit Expectation Gap in the United Kingdom", 1992.

19. Landslittle, David, in: "The Impact of Electronic Technology at the SEC", 1987.

20. The Auditing Practices Board of the Consultative Committee of Accountancy Bodies, "The Future Development of Auditing: A Paper to Promote Public Debate", November 1992.

21. Assurance Committee: "Future of the Financial Statement Audit", www.aicpa.org.

22. Assurance Committee: "Assurance Services - Definition and Interpretive Commentary", www.aicpa.org.

23. Assurance Committee: "Analysis of Interviews with Potential Assurance Customers", www.aicpa.org.

24. Power, Michael: „The Audit Society - Rituals of Verification", New York 1997. 